\title{
Light scattering by a magneto-optical nanoparticle in front of a flat surface: Perturbative approach
}

\author{
H. Marinchio, ${ }^{1}$ J. J. Sáenz, ${ }^{2}$ and R. Carminati ${ }^{1, *}$ \\ ${ }^{1}$ Institut Langevin, École Supérieure de Physique et de Chimie Industrielles de la Ville de Paris ParisTech, \\ Centre National de la Recherche Scientifique, 10 rue Vauquelin, 75231 Paris Cedex 05, France \\ ${ }^{2}$ Departamento de Física de la Materia Condensada, Universidad Autónoma de Madrid, 28049 Madrid, Spain
}

(Received 20 March 2012; published 13 June 2012)

\begin{abstract}
We develop a perturbative formalism for the interaction of a magneto-optical nanoparticle with a flat surface made of a dielectric or metallic material. The formalism leads to a simple interpretation of the interplay between the purely dielectric and the magneto-optical responses, in terms of excitation of (and radiation by) two orthogonal electric dipoles. We analyze two different routes for the enhancement of the magneto-optical response with respect to the purely dielectric contribution, both based on the nanoparticle-surface interaction. The enhancement is discussed in terms of relevant magneto-optical signals, such as changes in reflectivity, polarization (Kerr) rotation, and ellipticity.
\end{abstract}

DOI: 10.1103/PhysRevB.85.245425

PACS number(s): 78.67.-n, 78.20.Ls, 73.20.Mf

\section{INTRODUCTION}

The interaction between nanoscale objects (nanoparticles or nano-antennas) and dielectric or metallic surfaces is at the core of nano-optics and plasmonics. ${ }^{1-5}$ In the design of effective nanophotonic devices, the development of active components is a key issue. One aspect of active nanophotonics is the control of the optical response using an external parameter. ${ }^{6}$ Among various possible approaches, the use of a magneto-optical response, with the external static magnetic field as a control parameter, is receiving increasing attention. Recent studies have paved the way toward applications, such as nonreciprocal optical isolators ${ }^{7}$ and sensors, ${ }^{8}$ or have opened new perspectives, such as plasmonic interferometry, ${ }^{9}$ unidirectional plasmonic wave guiding, ${ }^{10}$ controlled molecular energy transfer, ${ }^{11}$ or random lasers. ${ }^{12}$ Magneto-optics is robust and offers several degrees of freedom since different type of signals can be exploited (e.g., changes in reflectivity or in polarization). But the weak magneto-optical response of usual materials (including ferromagnetics) needs to be compensated by an enhancement of light-matter interaction, usually from an enhancement of local electric fields. This can be done through a periodic molding of the magneto-optical material to create a photonic crystal. ${ }^{13}$ Another approach is to combine ferromagnetics and noble metals to take advantage of the large local electric field produced by the excitation of surface plasmons. ${ }^{14}$ This magnetoplasmonic enhancement has been demonstrated in multilayer structures ${ }^{15}$ and nanodisks or nanoholes arrays,${ }^{16}$ and seems to be a promising approach.

In this paper we examine theoretically the mechanisms of enhancement of the magneto-optical response of a nanoparticle interacting with a flat substrate. As a complementary alternative to numerical simulations, in Sec. II we introduce a perturbative approach that allows us to get a simple formalism offering a clear picture of the interaction. The perturbative treatment of the interaction between a particle made of an isotropic material and a flat surface has been used in pioneer studies of near-field optics. ${ }^{17}$ Here we develop a perturbative formalism that fully accounts for the anisotropic response of the nanoparticle, inherent to the magneto-optical response. In particular, we establish that under the assumption of a weak magneto-optical response (compared to the standard dielectric response), the induced electric dipole inside the nanoparticle can be written as the superposition of an isotropic dipole that describes the standard dielectric response and a magnetooptical dipole that describes the magneto-optical contribution. In the presence of a flat surface, it is known that the dressed polarizability of a nanoparticle can be enhanced by near-field interactions and surface-plasmon resonances. ${ }^{18,19}$ In the case of a magneto-optical nanoparticle, in Sec. III we show that the magneto-optical contribution in the dressed polarizability (driving the magneto-optical dipole) can be enhanced through the interaction with a flat surface, with respect to the purely dielectric contribution (driving the isotropic dipole). In the case of metallic surfaces, we identify the role of surfaceplasmon polaritons in the enhancement process. In Sec. IV we show that another way to enhance the magneto-optical signal is to optimize the radiation of the magneto-optical dipole, by choosing appropriate directions and polarizations of incidence and detection. We analyze the enhancement in terms of standard magneto-optical signals, such as changes in reflectivity, Kerr rotation, and ellipticity.

\section{PERTURBATIVE EXPRESSION OF THE ELECTRIC POLARIZABILITY}

In this section, we introduce the first-order perturbative formalism for the calculation of the polarizability of a small particle made of a magneto-optical (MO) material. We begin with the calculation of the quasistatic polarizability, and then derive the general expression of the dynamic effective polarizability.

\section{A. Magneto-optical response of a bulk material}

In the presence of a static external magnetic field, the dielectric function of an otherwise isotropic material becomes a tensor of the form ${ }^{20}$

$$
\boldsymbol{\epsilon}=\epsilon \mathbf{I}+\Delta \boldsymbol{\epsilon} \text { with } \Delta \boldsymbol{\epsilon}=\mathrm{i} Q \epsilon \mathbf{A},
$$

where $Q$ is the MO coefficient, $\epsilon$ the isotropic dielectric function in the absence of external magnetic field, and $\mathbf{I}$ the 
unit tensor. $\mathbf{A}$ is the following antisymmetric tensor:

$$
\mathbf{A}=\left(\begin{array}{ccc}
0 & -m_{z} & m_{y} \\
m_{z} & 0 & -m_{x} \\
-m_{y} & m_{x} & 0
\end{array}\right)
$$

written in a direct orthonormal reference frame, with $\boldsymbol{m}$ the unit vector in the direction of magnetization. In most cases, it is reasonable to assume that $|Q| \ll 1$, even for ferromagnetic materials (such as $\mathrm{Fe}, \mathrm{Ni}$, or $\mathrm{Co}$ ) that have relatively large MO responses. This means that in many situations MO effects can be treated in a perturbative approach, with $Q$ as a small parameter.

\section{B. Free-space quasistatic polarizability}

In the quasistatic regime, the free-space electric polarizability of a small (subwavelength) spherical particle made of an anisotropic material with dielectric tensor $\boldsymbol{\epsilon}$ is given by ${ }^{21}$ (see also the Appendix)

$$
\boldsymbol{\alpha}_{0}=3 V(\boldsymbol{\epsilon}-\mathbf{I})(\boldsymbol{\epsilon}+2 \mathbf{I})^{-1}
$$

with $V$ the volume of the particle. To first order in $|Q \epsilon| / \mid \epsilon+$ $2 \mid$, this becomes

$$
\begin{aligned}
\boldsymbol{\alpha}_{0} & =\frac{3 V}{\epsilon+2}(\boldsymbol{\epsilon}-\mathbf{I})\left(\mathbf{I}-\frac{\mathrm{i} Q \epsilon \mathbf{A}}{\epsilon+2}\right) \\
& =3 V \frac{\epsilon-1}{\epsilon+2}\left[\mathbf{I}+\frac{3 \mathrm{i} Q \epsilon \mathbf{A}}{(\epsilon+2)(\epsilon-1)}\right] .
\end{aligned}
$$

The polarizability is the sum of two terms, and can be written

$$
\boldsymbol{\alpha}_{0}=\boldsymbol{\alpha}_{0_{I}}+\boldsymbol{\alpha}_{0_{\mathrm{MO}}},
$$

where $\boldsymbol{\alpha}_{0_{I}}=\alpha_{0_{I}} \mathbf{I}$ is the quasistatic polarizability of a sphere with isotropic dielectric function $\epsilon$, where $\alpha_{0_{I}}=3 V(\epsilon-$ $1) /(\epsilon+2)$. The polarizability tensor $\alpha_{0_{\mathrm{MO}}}$ describes the MO response in the quasistatic limit. It can be written in the form $\boldsymbol{\alpha}_{0_{\mathrm{MO}}}=\delta \mathbf{A}$, where $\mathbf{A}$ is the dimensionless antisymmetric tensor introduced previously and $\delta=9 \mathrm{i} V Q \epsilon /(\epsilon+2)^{2}$ (the parameter $\delta$ will be used later for convenience). Even for ferromagnetic materials, the modulus of this term remains below one percent of $\left|\alpha_{0_{I}}\right|$ at any frequencies.

\section{Effective dynamic polarizability}

In the quasistatic regime, the interaction between the particle and the external field is fully described by the depolarization factor $(\boldsymbol{\epsilon}+2 \mathbf{I})^{-1}$ appearing in Eq. (3). In the dynamic regime, one has to account for the field radiated by the particle itself, which also influences the particle response (radiation reaction). The dipole moment at a frequency $\omega$ is then written as the product of the exciting field $\mathbf{E}_{0}$ and an effective polarizability $\boldsymbol{\alpha}_{\text {eff }}$

$$
\mathbf{p}=\epsilon_{0} \boldsymbol{\alpha}_{\text {eff }} \mathbf{E}_{0} .
$$

In the most general situation, the background medium can be inhomogeneous. We describe the electrodynamic response of the background (i.e., the environment of the polarizable particle) by the Green dyadic $\mathbf{G}\left(\mathbf{r}, \mathbf{r}^{\prime}, \omega\right)=\mathbf{G}_{0}\left(\mathbf{r}, \mathbf{r}^{\prime}, \omega\right)+\mathbf{S}\left(\mathbf{r}, \mathbf{r}^{\prime}, \omega\right)$, where $\mathbf{G}_{0}\left(\mathbf{r}, \mathbf{r}^{\prime}, \omega\right)$ is the free-space Green dyadic and $\mathbf{S}\left(\mathbf{r}, \mathbf{r}^{\prime}, \omega\right)$ describes the heterogeneities. In Eq. (6), the external field $\mathbf{E}_{0}$ has to be understood as the field in the background medium in the absence of the particle. In these conditions, the particle polarizability $\boldsymbol{\alpha}_{\text {eff }}$ is given by (see the Appendix for the full derivation)

$$
\boldsymbol{\alpha}_{\mathrm{eff}}=\left(\mathbf{I}-k^{2} \boldsymbol{\alpha}_{0} \mathbf{G}_{p}\right)^{-1} \boldsymbol{\alpha}_{0},
$$

where $k=\omega / c=2 \pi / \lambda$, with $c$ the speed of light in vacuum, $\omega$ the frequency of the exciting field, and $\lambda$ the wavelength. For the sake of clarity, we have introduced the interaction $\operatorname{dyadic} \mathbf{G}_{p}(\omega)=i \operatorname{Im}\left[\mathbf{G}_{0}\left(\mathbf{r}_{p}, \mathbf{r}_{p}, \omega\right)\right]+\mathbf{S}\left(\mathbf{r}_{p}, \mathbf{r}_{p}, \omega\right)$, where $\mathbf{r}_{p}$ is the position of the particle. The first term in $\mathbf{G}_{p}$ describes the free-space radiation reaction and the second term described the interaction with the structured environment. This form of the effective polarizability has been established previously in Ref. 22

In order to derive a first-order expression of the effective polarizability, we first rewrite $\boldsymbol{\alpha}_{\text {eff }}$ in the form

$$
\boldsymbol{\alpha}_{\mathrm{eff}}^{-1}=\boldsymbol{\alpha}_{0}^{-1}-k^{2} \mathbf{G}_{p} .
$$

Using Eq. (5), we obtain to first order in $|Q \epsilon| /|\epsilon+2|$

$$
\boldsymbol{\alpha}_{\mathrm{eff}}^{-1}=\alpha_{0_{I}}^{-1} \mathbf{I}-k^{2} \mathbf{G}_{p}-\boldsymbol{\Delta}_{\mathrm{MO}},
$$

where $\Delta_{\mathrm{MO}}=\alpha_{0_{I}}^{-2} \boldsymbol{\alpha}_{0_{\mathrm{MO}}}$. Assuming that $\alpha_{0_{I}}^{-1} \mathbf{I}-k^{2} \mathbf{G}_{p} \gg \boldsymbol{\Delta}_{\mathrm{MO}}$ (this inequality has to be understood in terms of the modulus of each component of the tensors), meaning that the magnetooptical contribution to the polarizability remains a weak correction, we obtain

$\boldsymbol{\alpha}_{\mathrm{eff}}=\left(\alpha_{0_{I}}^{-1} \mathbf{I}-k^{2} \mathbf{G}_{p}\right)^{-1}\left[\mathbf{I}+\left(\alpha_{0_{I}}^{-1} \mathbf{I}-k^{2} \mathbf{G}_{p}\right)^{-1} \boldsymbol{\Delta}_{\mathrm{MO}}\right]$.

This expression can be simplified by introducing the dyadic $\boldsymbol{D}_{p}=\left(\mathbf{I}-\alpha_{0_{I}} k^{2} \mathbf{G}_{p}\right)^{-1}$, which does not depend on the anisotropic response of the particle (this dyadic is not a perturbative quantity). We finally obtain a compact perturbative expression of the effective dynamic polarizability in an arbitrary environment

$$
\boldsymbol{\alpha}_{\text {eff }}=\boldsymbol{\alpha}_{0_{I}} \boldsymbol{D}_{p}+\boldsymbol{D}_{p} \boldsymbol{\alpha}_{0_{\mathrm{MO}}} \boldsymbol{D}_{p} .
$$

Note that in this expression the dyadic $\boldsymbol{D}_{p}$ accounts for the interaction between the isotropic response of the particle and the environment up to infinite order.

\section{Summary: Induced electric dipole}

Based on Eq. (11), we can write the induce dipole moment inside a MO particle as a sum of two terms

$$
\mathbf{p}=\epsilon_{0} \boldsymbol{\alpha}_{\mathrm{eff}} \mathbf{E}_{0}=\epsilon_{0}\left(\boldsymbol{\alpha}_{I}+\boldsymbol{\alpha}_{\mathrm{MO}}\right) \mathbf{E}_{0}=\mathbf{p}_{I}+\mathbf{p}_{\mathrm{MO}} .
$$

In this expression, we have introduced the polarizability $\boldsymbol{\alpha}_{I}=$ $\boldsymbol{\alpha}_{0_{I}} \boldsymbol{D}_{p}$ that describes the isotropic response (i.e., the response in absence of external magnetic field), and the polarizability $\boldsymbol{\alpha}_{\mathrm{MO}}=\boldsymbol{D}_{p} \boldsymbol{\alpha}_{0_{\mathrm{MO}}} \boldsymbol{D}_{p}$ that describes the MO response. The induced electric dipole $\mathbf{p}_{I}=\epsilon_{0} \boldsymbol{\alpha}_{I} \mathbf{E}_{0}$, corresponding to the isotropic response, will be denoted by isotropic dipole in the following. The induced electric dipole $\mathbf{p}_{\mathrm{MO}}=\epsilon_{0} \boldsymbol{\alpha}_{\mathrm{MO}} \mathbf{E}_{0}$ describes the perturbation induced by the magnetic field, and will be denoted by magneto-optical (MO) dipole. The perturbative approach developed above allows us to give a simple interpretation of the MO dipole: the electric field $\boldsymbol{D}_{p} \mathbf{E}_{0}$ due to the real exciting field $\mathbf{E}_{0}$ and the radiation of the isotropic dipole excites an anisotropic particle with polarizability $\boldsymbol{\alpha}_{\mathrm{MO}}$, which by self-radiation induces the MO 
dipole $\mathbf{p}_{\mathrm{MO}}=\epsilon_{0} \boldsymbol{D}_{p} \boldsymbol{\alpha}_{0_{\mathrm{MO}}} \boldsymbol{D}_{p}$. An important consequence is that the environment, whose influence is described by the tensor $\mathbf{G}_{p}$ (and consequently $\boldsymbol{D}_{p}$ ), modifies differently the isotropic and the anisotropic responses of the particle. This opens the possibility to relatively enhance the MO response by maximizing the ratio between the nondiagonal and the diagonal components of the effective polarizability taking advantage of favorable external configurations. In the following, we study the interaction between a single nanoparticle and a flat surface, in the framework of the perturbative analysis described above. We study the possibility of enhancing the MO response of the nanoparticle following two different strategies: enhancing the MO contribution in the dressed polarizability, or selecting preferentially the radiation of the induced MO dipole. Both strategies can be combined in practice.

\section{DRESSED POLARIZABILITY IN FRONT OF A FLAT SURFACE}

In this section, we analyze the dressed polarizability of a MO nanoparticle with radius $R$ located at a subwavelength distance $d \ll \lambda$ from a flat surface. Using the Green dyadic formalism automatically includes all interaction channels with the surface, including surface plasmons in the case of metallic surfaces. ${ }^{19}$ The surface separates free-space (medium $z>0$ ) from a semi-infinite metal (medium $z<0$ ) with dielectric function $\epsilon_{m}(\omega)$. We apply the first-order perturbative approach in order to estimate the influence of the surface on the dressed polarizability, and to identify a potential enhancement of the MO response with respect to the dielectric response. We assume that the dielectric properties of the metal substrate are not significantly affected by the presence of an external magnetic field, which is the case for noble metals. ${ }^{15}$ In contrast with MO garnets or ferromagnetic particles, the MO response of noble metals (typically three orders of magnitude weaker) is only relevant at very high magnetic fields. MO effects at relatively low static magnetic fields (1 Tesla) are relevant for some metal nanoparticles near the surface-plasmon resonance condition due to the large plasmon field enhancement. ${ }^{23}$

In the framework of the electric-dipole response (valid for $d>2 R$ ), we use the image dipole method to compute the Green dyadic, ${ }^{24}$ as sketched in Fig. 1. The dyadic $\mathbf{G}_{p}$ that drives the effective polarizability reads

$$
\begin{aligned}
\mathbf{G}_{p} \mathbf{p} & =i \operatorname{Im}\left[\mathbf{G}_{0}\left(\mathbf{r}_{p}, \mathbf{r}_{p}, \omega\right)\right] \mathbf{p}+\mathbf{S}\left(\mathbf{r}_{p}, \mathbf{r}_{p}, \omega\right) \mathbf{p} \\
& =i \operatorname{Im}\left[\mathbf{G}_{0}\left(\mathbf{r}_{p}, \mathbf{r}_{p}, \omega\right)\right] \mathbf{p}+\mathbf{G}_{0}\left(\mathbf{r}_{p}, \mathbf{r}_{p_{\mathrm{im}}}, \omega\right) \mathbf{p}_{\mathrm{im}} .
\end{aligned}
$$

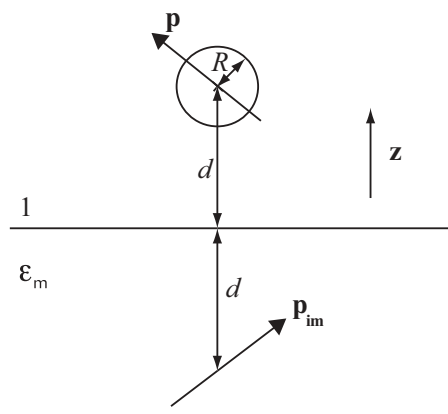

FIG. 1. Dipole-plane system. $\mathbf{p}$ is the induced dipole in the nanoparticle and $\mathbf{p}_{\mathrm{im}}$ is the image dipole.
In this expression, $\mathbf{G}_{0}$ is the free-space Green dyadic, and $\mathbf{p}_{\mathrm{im}}$ is the image dipole, located at position $\mathbf{r}_{p_{\text {im }}}$. In the short-distance regime $d \ll \lambda$, we can use the quasistatic approximation. The image dipole is given by

$$
\mathbf{p}_{\mathrm{im}}=\frac{\epsilon_{m}(\omega)-1}{\epsilon_{m}(\omega)+1}\left(\begin{array}{ccc}
-1 & 0 & 0 \\
0 & -1 & 0 \\
0 & 0 & 1
\end{array}\right) \mathbf{p} .
$$

The free-space Green dyadic $\mathbf{G}_{0}$ is real in this approximation, so that the term $i \operatorname{Im}\left[\mathbf{G}_{0}\left(\mathbf{r}_{p}, \mathbf{r}_{p}, \omega\right)\right]$ vanishes in the expression of $\mathbf{G}_{p}$. The surface contribution is given by

$$
\mathbf{G}_{0}\left(\mathbf{r}_{p}, \mathbf{r}_{p_{\mathrm{im}}}, \omega\right)=\frac{1}{4 \pi(2 d)^{3}}\left(\begin{array}{ccc}
-1 & 0 & 0 \\
0 & -1 & 0 \\
0 & 0 & 2
\end{array}\right)
$$

Finally, the perturbative expression of the effective polarizability in the quasistatic limit reads

$$
\begin{aligned}
& \boldsymbol{\alpha}_{\text {eff }}=\alpha_{0_{I}} \\
& \times\left(\begin{array}{ccc}
\frac{1}{1-\alpha_{0_{I}} \beta} & -\frac{m_{z} \delta}{\left(1-\alpha_{0_{I}} \beta\right)^{2}} & \frac{m_{y} \delta}{\left(1-\alpha_{0_{I}} \beta\right)\left(1-\alpha_{0_{I}} 2 \beta\right)} \\
\frac{m_{z} \delta}{\left(1-\alpha_{0_{I}} \beta\right)^{2}} & \frac{1}{1-\alpha_{0_{I}} \beta} & -\frac{m_{x} \delta}{\left(1-\alpha_{0_{I}} \beta\right)\left(1-\alpha_{0_{I}} 2 \beta\right)} \\
-\frac{m_{y} \delta}{\left(1-\alpha_{0_{I}} \beta\right)\left(1-\alpha_{0_{I}} 2 \beta\right)} & \frac{m_{x} \delta}{\left(1-\alpha_{0_{I}} \beta\right)\left(1-\alpha_{0_{I}} 2 \beta\right)} & \frac{1}{1-\alpha_{0_{I}} 2 \beta}
\end{array}\right)
\end{aligned}
$$

with $\beta(\omega, d)=\left[\epsilon_{m}(\omega)-1\right] /\left[32 \pi d^{3}\left(\epsilon_{m}(\omega)+1\right)\right]$.

Expression (16) shows that an exaltation of the MO effects can be obtained by minimizing the value of $1-\alpha_{0_{I}} \beta$ or $1-2 \alpha_{0_{I}} \beta$. In this case, the nondiagonal components of the polarizability can be increased, not only compared to their freespace values, but also compared to the diagonal components (one can increase the ratio $\left|\alpha_{\text {eff }}^{j i}\right| / \alpha_{\text {eff }}^{i i} \mid$ ). Since $\alpha_{0_{I}} \beta=\left[R^{3}(\epsilon-\right.$ 1) $\left(\epsilon_{m}-1\right) /\left[d^{3}(\epsilon+2)\left(\epsilon_{m}+1\right)\right]$ with $R<d / 2$, a significant enhancement can be expected for frequencies in the vicinity of plasmon resonances of either the particle or the surface. For the nanoparticle, the plasmon resonance occurs for $\operatorname{Re}[\epsilon(\omega)] \approx$ -2 , and is weak with magnetic materials due to substantial absorption. The plasmon resonance of the surface occurs for $\operatorname{Re}\left[\epsilon_{m}(\omega)\right] \approx-1$, and can play a role for a metal surface with low absorption. We show in Fig. 2 the polarizability of a Co sphere in the vicinity of an Ag or Au substrate. ${ }^{25}$ To maximize the dressed polarizability effect, we consider a distance $d=$ $2 R$ in the limit of validity of the dipole approximation. For the Ag surface, peaks around $\lambda=350 \mathrm{~nm}$ related to the plasmon resonance modify significantly the components involving $g_{z z}$ $\left(=2 g_{x x}=2 g_{y y}\right)$. No such resonances appear with the $\mathrm{Au}$ surface due to its higher absorption.

Finally let us comment on the use of the quasistatic approximation to compute the surface contribution to the effective polarizability. Since in this limit radiation by the particle is disregarded, energy conservation (or equivalently the optical theorem) is not satisfied. ${ }^{22,26}$ Nevertheless, it is important to point out that in the framework of the perturbation theory developed here, a first-order expansion of the effective polarizability in terms of the magneto-optical response $|Q \epsilon|$ excludes energy conservation in the first place. Indeed, the optical theorem necessarily combines first-order and secondorder terms (proportional to $\boldsymbol{\alpha}_{\text {eff }}$ and $\left|\boldsymbol{\alpha}_{\text {eff }}\right|^{2}$ ). Therefore, a 

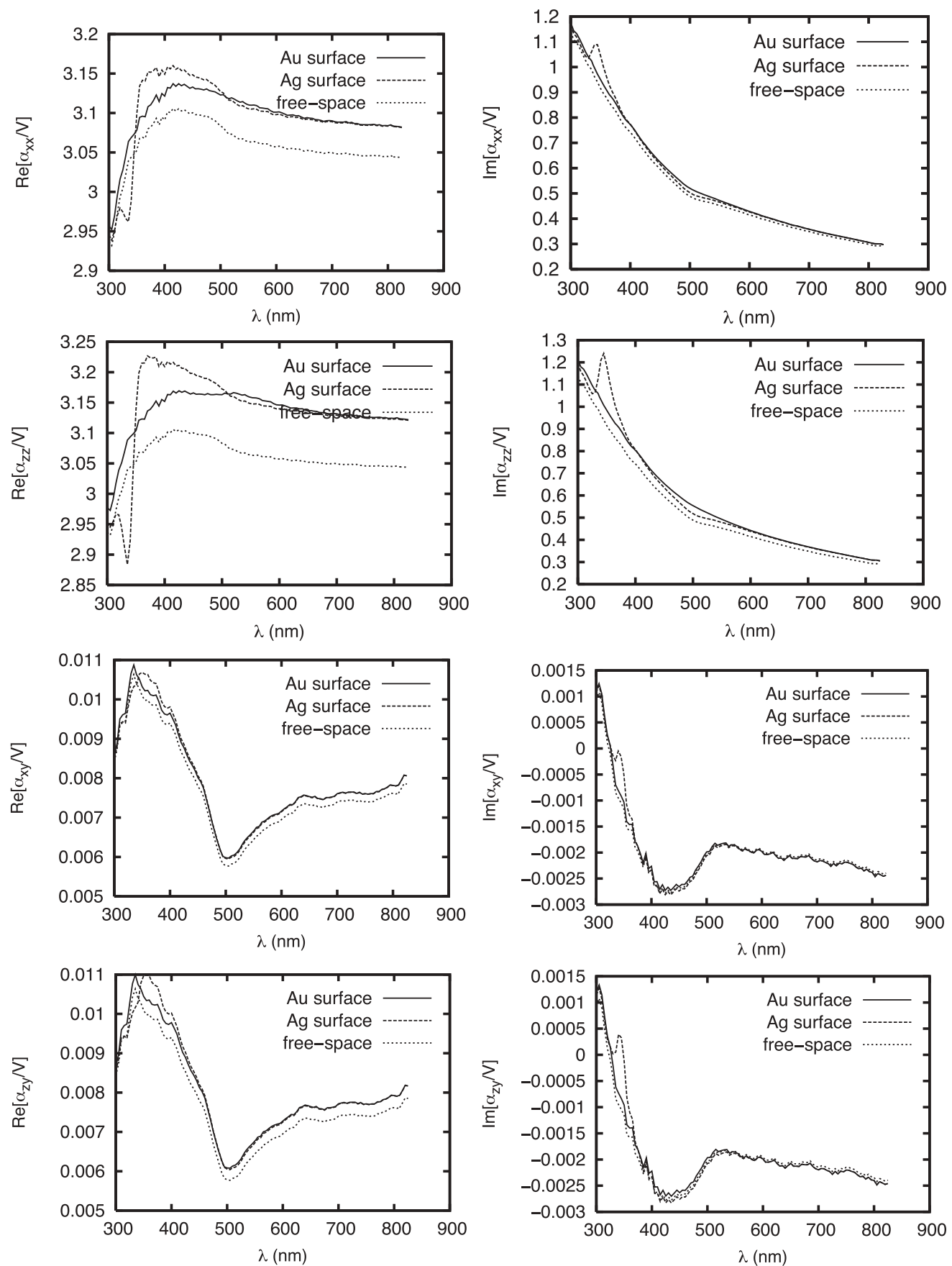

FIG. 2. Diagonal and nondiagonal components of the effective polarizability tensor of a 10-nm radius cobalt sphere standing $20 \mathrm{~nm}$ above a metal surface (normalized by the volume of the nanoparticle). For the calculation of $\alpha_{x y}\left(\alpha_{z y}\right)$, we assume a static external magnetic field oriented along the $z(x)$ direction. The dielectric function of $\mathrm{Au}, \mathrm{Ag}$, and Co is taken from. ${ }^{25}$

correct treatment of energy conservation would require not only a computation of the surface contribution including retardation, but a treatment beyond first-order perturbation. In practical terms, this means that the approach developed here leads to accurate computations of fields, which permits the computation of several magneto-optical signals of practical interest (such as polarization rotation or ellipticity). But it might be inappropriate for the computations of intensities or radiative fluxes.

\section{DIFFRACTION OF A PLANE WAVE BY A MAGNETO-OPTICAL NANOPARTICLE-SUBSTRATE SYSTEM}

In this section we examine another strategy to enhance the MO contribution in the field scattered by a MO nanoparticle, compared to the standard dielectric response. The idea is to select preferentially the emission of the MO induced dipole, taking advantage of the fact that it is perpendicular to the dielectric (isotropic) induced dipole. We will see that this 


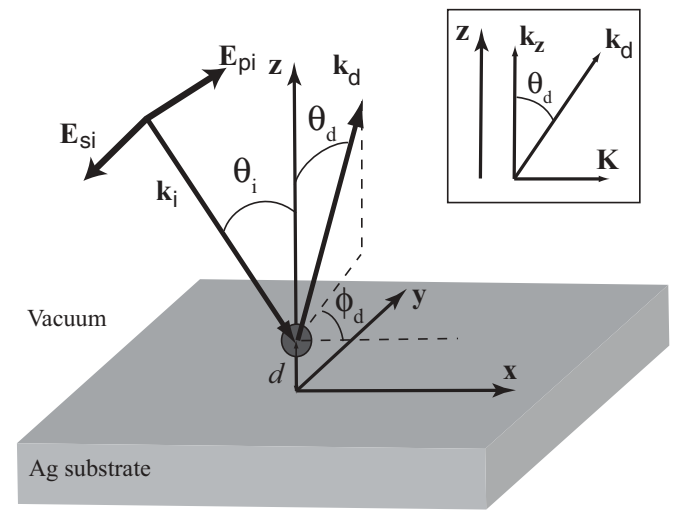

FIG. 3. MO nanoparticle in the vicinity of a flat interface between vacuum and a semi-infinite medium with dielectric function $\epsilon_{m}(\omega)$. The incidence plane is $(\hat{\mathbf{x}}, \hat{\mathbf{z}}) ; \theta_{i}$ is the incidence angle while $\theta_{d}$ and $\phi_{d}$ correspond the observation direction. $\mathbf{k}_{i}$ and $\mathbf{k}_{d}$ represent the incoming and the diffracted wave vectors (corresponding to the observation angles $\theta_{d}$ and $\phi_{d}$ ). $\mathbf{K}=K \hat{\boldsymbol{K}}=k \sin \theta_{d} \hat{\boldsymbol{K}}$ and $\mathbf{k}_{z}=$ $\sqrt{k^{2}-K^{2}} \hat{\mathbf{z}}=k \cos \theta_{d} \hat{\mathbf{z}}$ are the components of the diffracted wave vector parallel and normal to the metal surface.

selection can be efficient in the presence of a flat surface, that stimulates the polarization selectivity.

\section{A. General expression of the diffracted far field}

As in the previous section, we consider a nanoparticle with radius $R \ll \lambda$, located in vacuum at a distance $d$ above a flat substrate with dielectric function $\epsilon_{m}(\omega)$. The geometry is sketched in Fig. 3. In order to focus on the radiation selection process, we do not account here for the surface effect on the dressed polarizability and use the bare quasistatic polarizability. In terms of the exciting field $\mathbf{E}_{e}$, the induced electric dipole reads

$$
\mathbf{p}=\mathbf{p}_{I}+\mathbf{p}_{\mathrm{MO}}=\epsilon_{0} \boldsymbol{\alpha}_{0_{I}} \mathbf{E}_{e}+\epsilon_{0} \boldsymbol{\alpha}_{0_{\mathrm{MO}}} \mathbf{E}_{e} .
$$

Note that using the effective (dressed) polarizability would not change anything at this stage. The far field radiated at position $\mathbf{r}$ by the induced dipole (with $|\mathbf{r}|=r \gg \lambda$ ) reads

$$
\mathbf{E}_{d}(\mathbf{r})=\frac{k^{2} \exp (\mathrm{i} k r)}{4 \pi \epsilon_{0} r} \mathbf{R}(\mathbf{K}) \mathbf{p},
$$

where $\mathbf{K}$ is the component of the diffracted wave vector along the plane parallel to the surface. The tensor $\mathbf{R}(\mathbf{K})$ described both the free-space and the reflected radiations and is given by

$$
\begin{aligned}
\mathbf{R}(\mathbf{K})= & {\left[\left(\hat{\mathbf{s}} r^{s}\left(\theta_{d}\right) \hat{\mathbf{s}}+\hat{\mathbf{p}}^{+} r^{p}\left(\theta_{d}\right) \hat{\mathbf{p}}^{-}\right) \exp \left(2 \mathrm{i} k \cos \theta_{d} d\right)\right.} \\
& \left.+\left(\hat{\mathbf{s}} \hat{\mathbf{S}}+\hat{\mathbf{p}}^{+} \hat{\mathbf{p}}^{+}\right)\right] .
\end{aligned}
$$

In this expression, $r^{s}\left(\theta_{d}\right)$ and $r^{p}\left(\theta_{d}\right)$ are the amplitude Fresnel reflection factors for $s$ and $p$ polarizations, respectively. Following the notations in Ref. 27, we have used the unit vectors $\hat{\mathbf{s}}, \hat{\mathbf{p}}^{+}$, and $\hat{\mathbf{p}}^{-}$that define the directions of the electric field in $s$ and $p$ polarizations (for $p$ polarization, the electric field has two different directions for upward and downward plane waves, identified with the superscript + and - ). They are given by

$$
\begin{aligned}
\hat{\mathbf{s}} & =\hat{\boldsymbol{K}} \times \hat{\mathbf{z}} \\
\hat{\mathbf{p}}^{+} & =(K / k) \hat{\mathbf{z}}-\left(k_{z} / k\right) \hat{\boldsymbol{K}}=\sin \theta_{d} \hat{\mathbf{z}}-\cos \theta_{d} \hat{\boldsymbol{K}} \\
\hat{\mathbf{p}}^{-} & =(K / k) \hat{\mathbf{z}}+\left(k_{z} / k\right) \hat{\boldsymbol{K}}=\sin \theta_{d} \hat{\mathbf{z}}+\cos \theta_{d} \hat{\boldsymbol{K}},
\end{aligned}
$$

where the symbol^ denotes a unit vector. Using Eqs. (17) and (18), the scattered far field can be split into two parts

$$
\mathbf{E}_{d}=\left[\mathbf{E}_{d}^{I}(\mathbf{K})+\mathbf{E}_{d}^{\mathrm{MO}}(\mathbf{K})\right] \frac{\exp (\mathrm{i} k r)}{4 \pi r}
$$
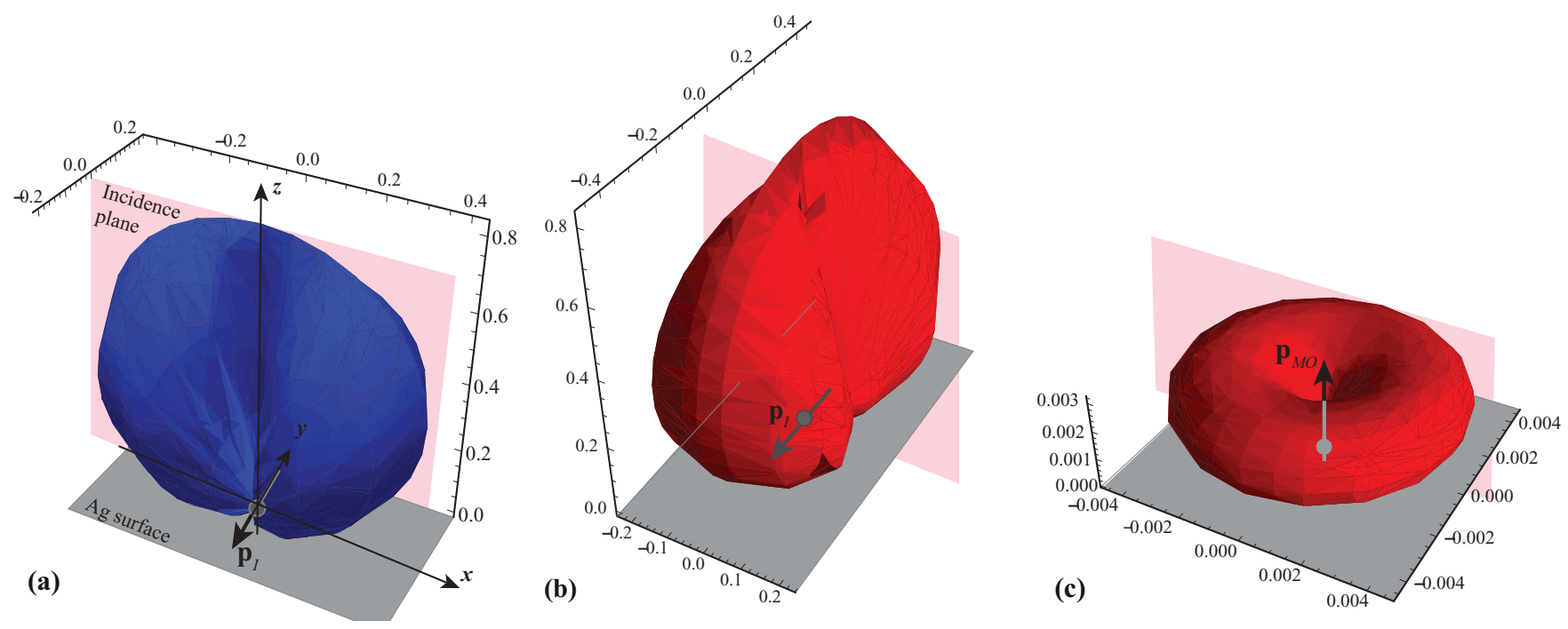

FIG. 4. (Color online) Spherical plot of the modulus of the far-field amplitude of the diffracted electric field $\mathbf{E}_{d}\left(\theta_{d}, \phi_{d}\right)$. The observation angles $\theta_{d}$ and $\phi_{d}$ are defined in Fig. 3. Three different contributions are represented: (a) the $s$ field radiated by the isotropic dipole, (b) the $p$ field radiated by the isotropic dipole, and (c) the $p$ field radiated by the MO dipole. The far-field amplitudes are normalized by $k^{2} \alpha_{0_{I}} E_{s_{i}} \exp (\mathrm{i} k r) /(4 \pi r)$, which represents the amplitude of the scattered field in the plane of incidence in vacuum and in the absence of magnetic field. We have considered a Co particle with radius $R=10 \mathrm{~nm}$ at a distance $d=20 \mathrm{~nm}$ above an Ag surface illuminated by an $s$-polarized plane wave at normal incidence. $\lambda=630 \mathrm{~nm}$. The arrows represent the emitting induced dipoles: the isotropic dipole on (a) and (b); the MO dipole on (c). 
with

$$
\begin{aligned}
\mathbf{E}_{d}^{I}(\mathbf{K}) & =k^{2} \mathbf{R}(\mathbf{K}) \boldsymbol{\alpha}_{0_{I}} \mathbf{E}_{e} \\
\mathbf{E}_{d}^{\mathrm{MO}}(\mathbf{K}) & =k^{2} \mathbf{R}(\mathbf{K}) \boldsymbol{\alpha}_{0_{\mathrm{MO}}} \mathbf{E}_{e} .
\end{aligned}
$$

The field radiated by the MO-induced dipole (referred to as the MO field) $\mathbf{E}_{d}^{\mathrm{MO}}$ only appears in the presence of the external magnetic field. The field radiated by the isotropic dipole $\mathbf{E}_{d}^{I}$ does not depend on the applied magnetic field. These two components can be calculated separately, and their relative weight can be analyzed.

For the sake of illustration, let us consider a situation in which the external magnetic field is applied along the $x$ axis ( $\boldsymbol{M}=\boldsymbol{M} \hat{\mathbf{x}}$, longitudinal configuration), and the exciting field is $s$ polarized. This yields

$$
\mathbf{E}_{e}=-E_{S_{i}}\left[1+r^{s}\left(\theta_{i}\right) \exp \left(2 \mathrm{i} k \cos \theta_{i} d\right)\right] \hat{\mathbf{y}} .
$$

Using Eq. (22), we can calculate the amplitude of the diffracted fields $\mathbf{E}_{d}^{\mathrm{MO}}$ and $\mathbf{E}_{d}^{I}$. Their normalized moduli are represented in Fig. 4 versus the observation angles $\theta_{d}$ and $\phi_{d}$, for an exciting field at normal incidence. The two excited dipoles being orthogonal, their $p$ and $s$ radiation patterns are significantly different. This can be used to extract selectively the MO contribution. Indeed, in this case, an $s-p$ conversion occurs since the MO dipole is normal to the surface, while the isotropic dipole remains parallel to the surface. In particular, for an observation in the plane of incidence $\left(\phi_{d}=0\right)$, the scattered $p$ waves are only produced by the MO dipole.
Therefore a $p$-polarized detection in this plane would extract the $\mathrm{MO}$ response of the nanoparticle from the overall response.

\section{B. Particular case: Diffraction in the plane of incidence}

It is instructive to study more carefully the diffracted field in the plane of incidence $\left(\phi_{d}=0\right)$. We will study separately the $s$-polarized and $p$-polarized excitations.

\section{1. s-polarized incident wave}

(i) For $\boldsymbol{M}=M \hat{x}$ ( longitudinal configuration), as already seen in the previous subsection, the magnetic field induces a MO dipole in the incidence plane, which radiates $p$ waves [see Fig. 5 (a)]. In the incidence plane, the contribution of the MO dipole can be easily extracted since it is orthogonal to the $s$-polarized isotropic field. A figure of merit is the ratio between the amplitudes of the radiated MO field in presence of the surface and in absence of the surface. It can be expressed using (22) and (23)

$$
\begin{aligned}
\gamma= & \frac{\left|\mathbf{E}_{\mathrm{d}}^{\mathrm{MO}}\right|}{\left|\mathbf{E}_{d \text { free-space }}^{\mathrm{MO}}\right|}=\left|1+r^{s}\left(\theta_{i}\right) \exp \left(2 \mathrm{i} k \cos \theta_{i} d\right)\right| \\
& \times\left|1+r^{p}\left(\theta_{d}\right) \exp \left(2 \mathrm{i} k \cos \theta_{d} d\right)\right| .
\end{aligned}
$$

The first factor is related to the reflection of the $s$-polarized incident wave and involves the reflection coefficient $r^{s}$ while the second corresponds to the reflection of the diffracted $p$
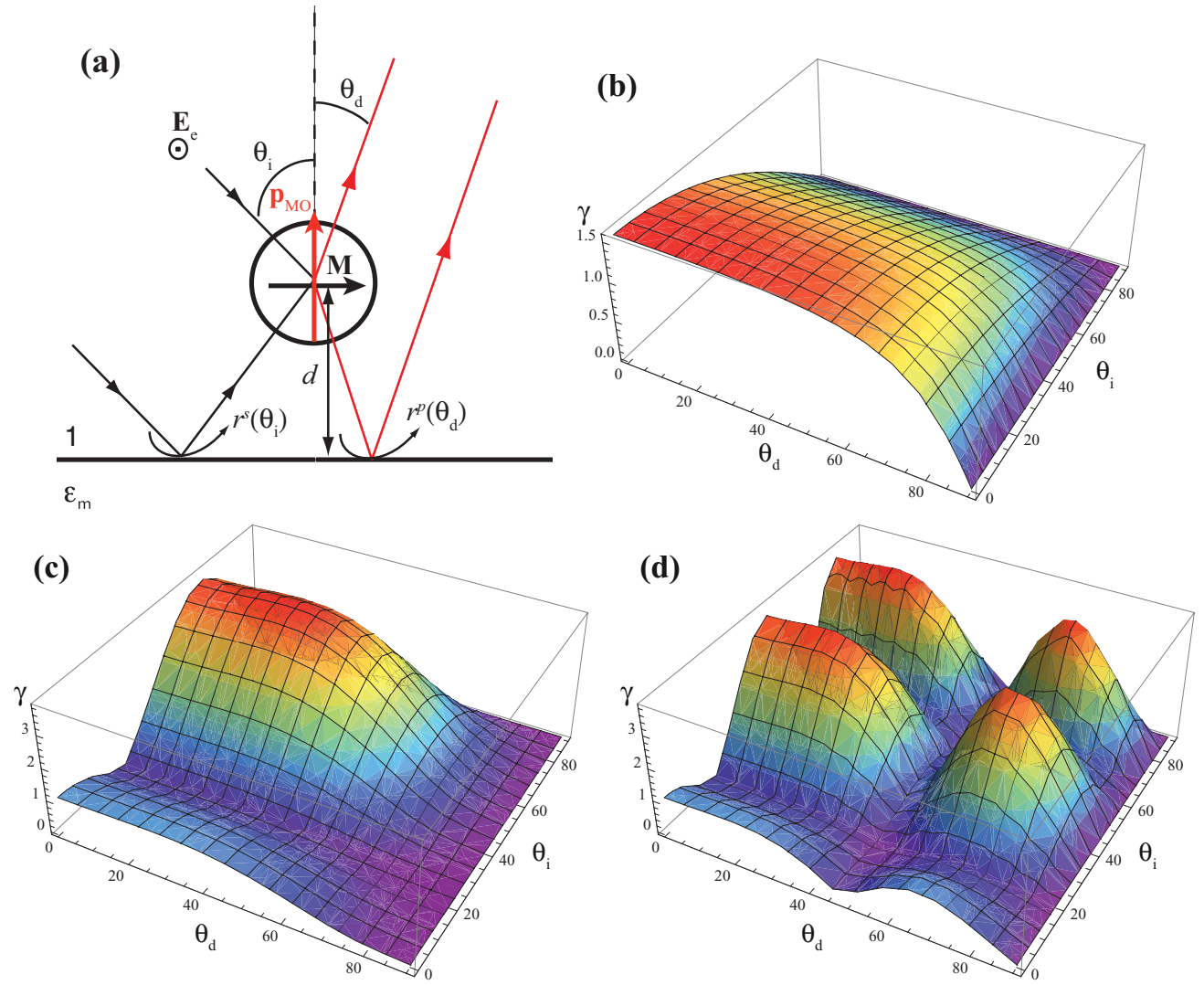

FIG. 5. (Color online) (a) Schematic representation of the MO dipole radiation when the exciting field is $s$ polarized and with $\boldsymbol{M}=M \hat{\mathbf{x}}$. (b) Figure of merit $\gamma$ versus incidence and observation angles for a Co sphere with radius $R=10 \mathrm{~nm}$ above an Ag substrate for different particle-plane distance $d$ : (b) $d=20 \mathrm{~nm}$; (c) $d=\lambda / 2$; (d) $d=\lambda$. Illumination wavelength $\lambda=630 \mathrm{~nm}$. 

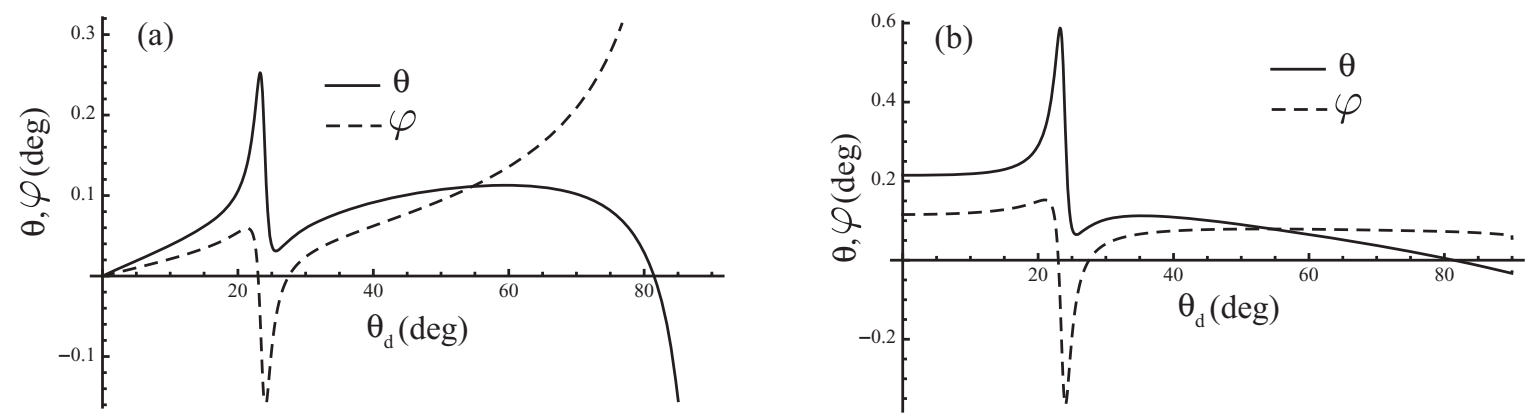

FIG. 6. Real Kerr rotation $\theta$ and ellipticity $\varphi$ of the scattered field versus the observation angle ( $s$ polarization). (a): $\boldsymbol{M}=M \hat{x}$, (b): $\boldsymbol{M}=M \hat{z}$. $d=\lambda / 2=315 \mathrm{~nm}$.

waves. We describe here an unusual mirror effect, which involves reflection of both $s$ - and $p$-polarized waves.

We show in Fig. 5 the ratio $\gamma$ versus the incidence and observation angles. The result demonstrates that the radiation of the MO dipole can be enhanced by the presence of a metal surface. Indeed, when the interferences between the reflected and nonreflected waves are constructive, the amplitude of the MO scattered is almost multiplied by a factor of four (both incoming and scattered waves being almost multiplied by a factor of two due to reflection) with respect to its value in free space.

Another useful feature of the MO response is the complex Kerr rotation (MOKE) $\Phi=\theta+\mathrm{i} \varphi$, where $\theta$ is the real Kerr rotation and $\varphi$ the ellipticity. For weak anisotropy (assumed in the perturbative approach), it can be accurately approximated by the ratio between the MO field ( $p$ polarized) and the isotropic field ( $s$ polarized). ${ }^{22}$ In the longitudinal configuration we then have

$\Phi_{\mathrm{LMOKE}}^{(s)} \simeq \frac{\mathbf{E}_{d}^{\mathrm{MO}} \cdot \hat{\mathbf{p}}^{+}}{\mathbf{E}_{d}^{I} \cdot \hat{\mathbf{s}}}=\frac{\delta \sin \theta_{d}\left[1+r^{p}\left(\theta_{d}\right) \exp \left(2 \mathrm{i} k \cos \theta_{d} d\right)\right]}{\alpha_{0_{I}}\left[1+r^{s}\left(\theta_{d}\right) \exp \left(2 \mathrm{i} k \cos \theta_{d} d\right)\right]}$

(ii) For $\boldsymbol{M}=\boldsymbol{M \hat { \mathbf { y } }}$ (transversal configuration), the collinearity of the static magnetic field and the exciting electric field prevents any MO response to be effective.

(iii) For $\boldsymbol{M}=M \hat{z}$ (polar configuration), an $s-p$ conversion also occurs. Indeed, the incoming radiation induces a MO dipole along the $x$ direction, lying in the incidence plane. The

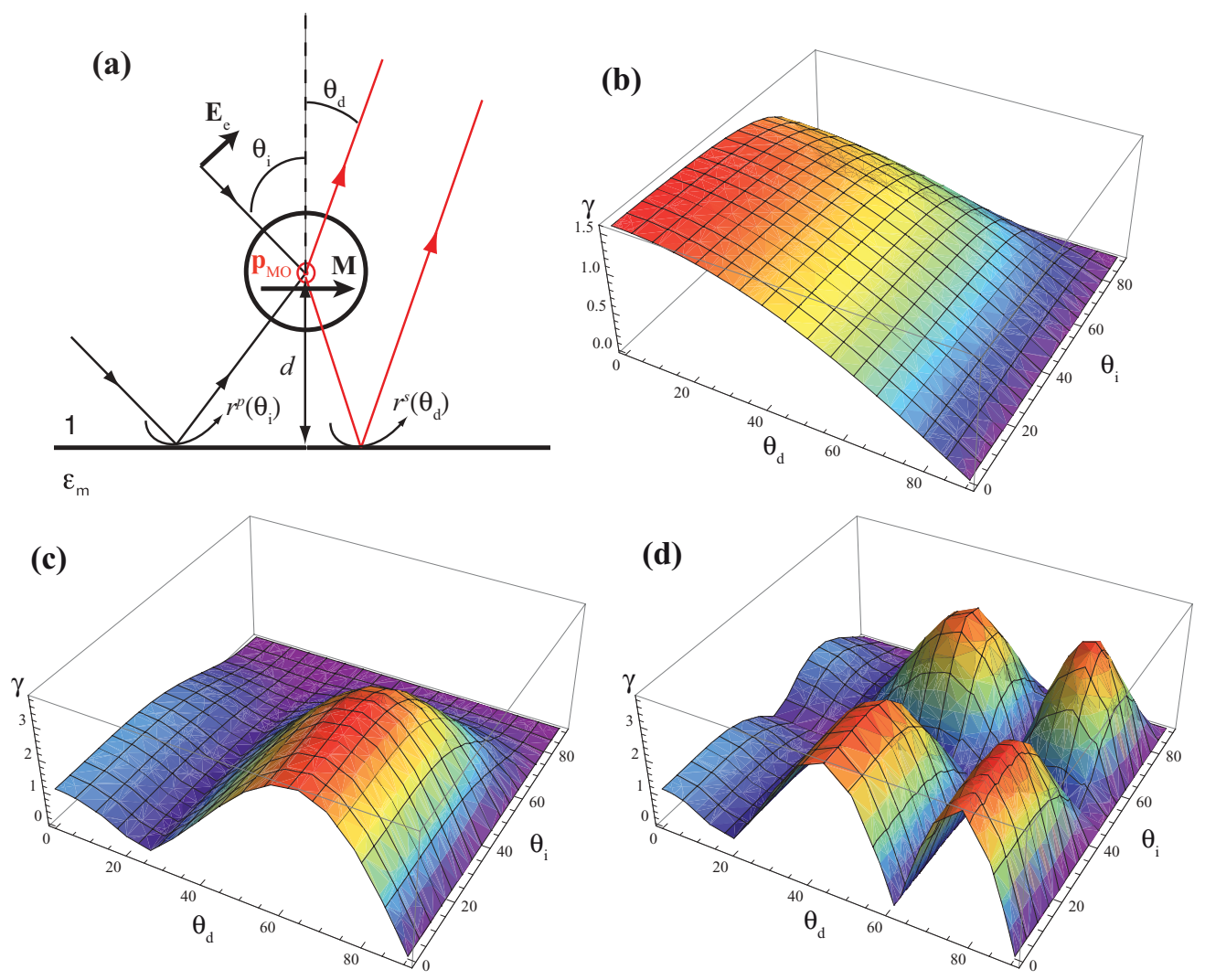

FIG. 7. (Color online) Same as Fig. 5 but with a $p$-polarized incident field. 
figure of merit $\gamma$ reads in this case

$$
\begin{aligned}
\gamma= & \frac{\left|\mathbf{E}_{\mathrm{d}}^{\mathrm{MO}}\right|}{\left|\mathbf{E}_{d \text { free-space }}^{\mathrm{MO}}\right|}=\left|1+r^{s}\left(\theta_{i}\right) \exp \left(2 \mathrm{i} k \cos \theta_{i} d\right)\right| \\
& \times\left|1-r^{p}\left(\theta_{d}\right) \exp \left(2 \mathrm{i} k \cos \theta_{d} d\right)\right| .
\end{aligned}
$$

The complex Kerr rotation can also be calculated

$$
\Phi_{\mathrm{PMOKE}}^{(s)} \simeq \frac{\delta \cos \theta_{d}\left[1-r^{p}\left(\theta_{d}\right) \exp \left(2 \mathrm{i} k \cos \theta_{d} d\right)\right]}{\alpha_{0_{I}}\left[1+r^{s}\left(\theta_{d}\right) \exp \left(2 \mathrm{i} k \cos \theta_{d} d\right)\right]} .
$$

It is worth noticing that, for $s$-polarized waves, the MOKE signal from a single particle does not depend on the angle of incidence since both isotropic and MO-induced dipoles are not modified by this parameter. The real Kerr rotation $\theta$ and the ellipticity $\varphi$ are represented in Fig. 6 versus the observation angle $\theta_{d}$, for both magnetization directions ( $\boldsymbol{M}=$ $M \hat{x}$ and $\boldsymbol{M}=M \hat{z})$. The important increase of both angles for $\theta_{d} \approx 20-25^{\circ}$ is related to the weakening of the isotropic field due to destructive interferences. The rapid change in the Kerr rotation could be useful to detect small changes in the particle's environment, which could be of interest from the point of view of single-particle, MO-based chemical sensors.

\section{2. p-polarized incident wave}

For an illumination in $p$ polarization, the incident field reads

$$
\begin{aligned}
\mathbf{E}_{e}= & E_{p_{i}} \cos \theta_{i}\left[1-r^{p}(\alpha) \exp \left(2 \mathrm{i} k \cos \theta_{i} d\right)\right] \hat{\mathbf{x}} \\
& +E_{p_{i}} \sin \theta_{i}\left[1+r^{p}\left(\theta_{i}\right) \exp \left(2 \mathrm{i} k \cos \theta_{i} d\right)\right] \hat{\mathbf{z}} .
\end{aligned}
$$

(i) For $\boldsymbol{M}=M \hat{\mathbf{x}}$, the $\mathrm{MO}$ response generates a $p-s$ conversion. The component of the exciting field normal to the surface induces a MO dipole that radiates $s$ waves [see Fig. 7(a)]. The figure of merit $\gamma$ is given by

$$
\begin{aligned}
\gamma= & \frac{\left|\mathbf{E}_{\mathrm{d}}^{\mathrm{MO}}\right|}{\left|\mathbf{E}_{d \text { free-space }}^{\mathrm{MO}}\right|}=\left|1+r^{p}\left(\theta_{i}\right) \exp \left(2 \mathrm{i} k \cos \theta_{i} d\right)\right| \\
& \times\left|1+r^{s}\left(\theta_{d}\right) \exp \left(2 \mathrm{i} k \cos \theta_{d} d\right)\right| .
\end{aligned}
$$

Therefore, as it can be checked in Fig. 7, the situation is reciprocal to the case of the $s-p$ conversion shown previously in Fig. 5. A mirror effect can also be exploited to radiate a stronger MO field.

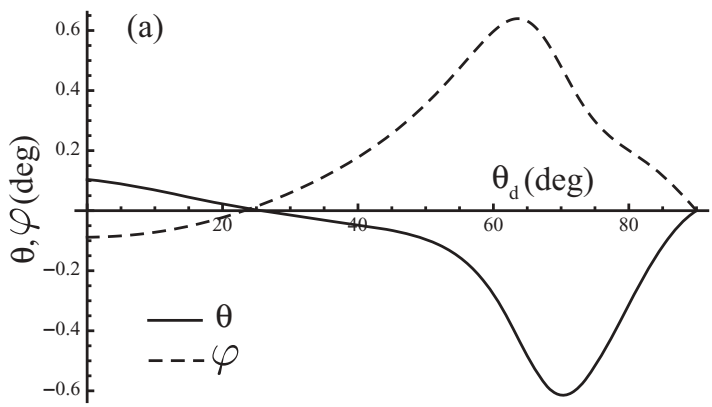

The complex Kerr rotation reads in this case

$$
\begin{aligned}
& \Phi_{\mathrm{LMOKE}}^{(p)} \approx \frac{\mathbf{E}_{d}^{\mathrm{MO}} \cdot \hat{\mathbf{s}}}{\mathbf{E}_{d}^{I} \cdot \hat{\mathbf{p}}^{+}} \\
& =\frac{\delta \sin \theta_{i}\left[1+r^{s}\left(\theta_{d}\right) \exp \left(2 \mathrm{i} k \cos \theta_{d} d\right)\right]}{\alpha_{0_{I}}\left[\sin \left(\theta_{i}-\theta_{d}\right)+\cos \left(\theta_{i}-\theta_{d}\right) r^{p}\left(\theta_{d}\right) \exp \left(2 \mathrm{i} k \cos \theta_{d} d\right)\right]} .
\end{aligned}
$$

(ii) For $\boldsymbol{M}=M \hat{\mathbf{y}}$, the $\mathrm{MO}$ effect induces a rotation but the MO dipole remains in the incidence plane. Since the fields radiated by the MO and isotropic dipoles are not orthogonal, this situation is not appropriate for an efficient selection of the MO contribution.

(iii) For $\boldsymbol{M}=\boldsymbol{M} \hat{\mathbf{z}}$, the phenomenon is similar to that observed for $\boldsymbol{M}=\boldsymbol{M} \hat{\mathbf{x}}$. The difference is that in the present case the MO dipole is induced by the $x$ component of the field. This yields the following figure of merit:

$$
\begin{aligned}
\gamma= & \frac{\left|\mathbf{E}_{\mathrm{d}}^{\mathrm{MO}}\right|}{\left|\mathbf{E}_{d \text { free-space }}^{\mathrm{MO}}\right|}=\left|1-r^{p}\left(\theta_{i}\right) \exp \left(2 \mathrm{i} k \cos \theta_{i} d\right)\right| \\
& \times\left|1+r^{s}\left(\theta_{d}\right) \exp \left(2 \mathrm{i} k \cos \theta_{d} d\right)\right| .
\end{aligned}
$$

Similarly, the complex Kerr rotation reads

$$
\begin{aligned}
& \Phi_{\mathrm{PMOKE}}^{(p)} \\
& \quad=\frac{\delta \cos \theta_{i}\left[1+r^{s}\left(\theta_{d}\right) \exp \left(2 \mathrm{i} k \cos \theta_{d} d\right)\right]}{\alpha_{0_{I}}\left[\sin \left(\theta_{i}-\theta_{d}\right)+\cos \left(\theta_{i}-\theta_{d}\right) r^{p}\left(\theta_{d}\right) \exp \left(2 \mathrm{i} k \cos \theta_{d} d\right)\right]} .
\end{aligned}
$$

The real Kerr rotation $\theta$ and the ellipticity $\varphi$ are represented in Fig. 8 versus the observation angle $\theta_{d}$, for these two configurations: (a) $\boldsymbol{M}=M \hat{x}$ at grazing incidence and (b) $\boldsymbol{M}=$ $M \hat{z}$ at normal incidence. On the one hand, both configurations yield to the same (maximized) MO dipole in the $\hat{\mathbf{y}}$ direction. The increase of the Kerr effect for $\theta_{d}$ is mainly due to the constructive interferences between the reflected and direct scattered $s$ waves [also seen on Fig. 7(c) for $\theta_{i}=0$ ] radiated by the MO dipole. On the other hand, the isotropic dipole is modified: it is normal to the surface in the first case (a) and in the $\hat{\mathbf{x}}$ direction in the second one (b). Thus, the differences between the two subfigures in Fig. 8 are only due to the radiation of the isotropic dipoles.

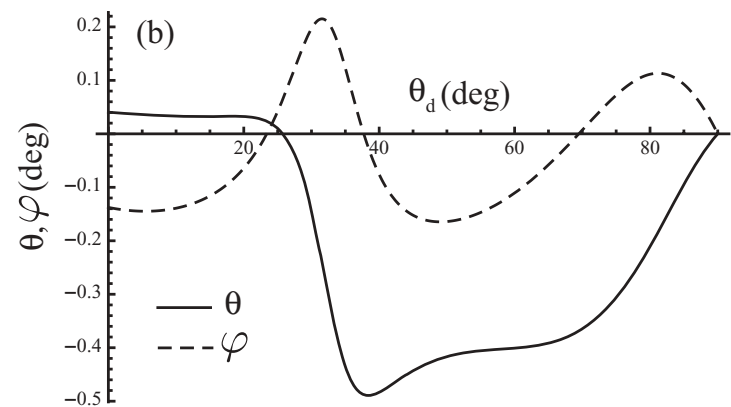

FIG. 8. Real Kerr rotation $\theta$ and ellipticity $\varphi$ of the scattered field versus the observation angle $\theta_{d}$ ( $p$ polarization). (a): $\boldsymbol{M}=M \hat{x}$ and $\theta_{i}=90^{\circ}$. (b): $\boldsymbol{M}=M \hat{z}$ and $\theta_{i}=0 . d=\lambda / 2=315 \mathrm{~nm}$. 


\section{CONCLUSION}

In conclusion, we have developed a perturbative formalism for the interaction of a magneto-optical nanoparticle with a flat surface made of a dielectric or metallic material. The nanoparticle has been assumed to be electrically small, and treated in the electric dipole approximation. We have shown that under the assumption of a weak magneto-optical response (compared to the standard dielectric response), the polarizability can be written as the sum of an isotropic polarizability, that describes the standard dielectric response, and a magneto-optical polarizability that describes the magnetooptical contribution. This point of view leads to a simple interpretation of the interplay between the purely dielectric and the magneto-optical responses, in terms of excitation of (and radiation by) two orthogonal electric dipoles. Such an approach is efficient for the analysis of complex geometries (e.g., prior to full numerical simulations) and for the qualitative interpretation of experimental results, since it permits a direct computation of standard magneto-optical signals such as changes in reflectivity (or scattering cross sections), polarization rotations, and ellipticity. In the presence of a flat surface, the magneto-optical contribution in the dressed polarizability can be enhanced with respect to the purely dielectric contribution due to near-field interactions, the enhancement process being reinforced by the excitation of surface-plasmon resonances in the case of a metallic surface. The anisotropic response of the nanoparticles-substrate system also provides another possibility for the enhancement of the magneto-optical contribution to the light scattered by the nanoparticle (with respect to the purely dielectric contribution), by selecting the directions and polarizations of incidence and detection. Future work might focus on more refined analysis of the interaction with surface-plasmon polaritons (e.g., the possibility of a local excitation mediated by the magneto-optical response of a nanoparticle) and/or on the magneto-optical response of an ensemble of nanoparticles forming a periodic array or a disordered medium.

\section{ACKNOWLEDGMENT}

This work was supported by the EU Project Nanomagma NMP3-SL-2008-214107.

\section{APPENDIX: EXPRESSION OF THE EFFECTIVE POLARIZABILITY}

Some general properties of the effective polarizability tensor have been discussed in Ref. 22. For completeness, we deduce here the expression of the effective polarizability consistent with the notation followed in the present work. We consider a homogeneous and small spherical particle occupying a volume $V$ around the position $\mathbf{r}_{p}$. The radius of the sphere $R$ is weak in front of the wavelength and its dielectric tensor is noted $\boldsymbol{\epsilon}(\omega)$.

The total field $\mathbf{E}(\mathbf{r})$ at the position $\mathbf{r}$ can be expressed as the sum of the exciting field $\mathbf{E}_{0}(\mathbf{r})$ and the field radiated by the particle :

$$
\mathbf{E}(\mathbf{r})=\mathbf{E}_{0}(\mathbf{r})+k^{2} \int_{V} \mathbf{G}\left(\mathbf{r}, \mathbf{r}^{\prime}, \omega\right) \epsilon_{0}(\boldsymbol{\epsilon}-\mathbf{I}) \mathbf{E}\left(\mathbf{r}^{\prime}\right) d^{3} \mathbf{r}^{\prime}
$$

We introduce here $\mathbf{G}\left(\mathbf{r}, \mathbf{r}^{\prime}, \omega\right)$ is the Green tensor describing the propagation in the external medium between one source point at $\mathbf{r}$ and a target point at $\mathbf{r}^{\prime}$. One can separate this tensor in two contributions $\mathbf{G}\left(\mathbf{r}, \mathbf{r}^{\prime}, \omega\right)=\mathbf{G}_{0}\left(\mathbf{r}, \mathbf{r}^{\prime}, \omega\right)+\mathbf{S}\left(\mathbf{r}, \mathbf{r}^{\prime}, \omega\right) . \mathbf{G}_{0}\left(\mathbf{r}, \mathbf{r}^{\prime}, \omega\right)$ corresponds to free-space radiation and $\mathbf{S}\left(\mathbf{r}, \mathbf{r}^{\prime}, \omega\right)$ is due to external scatterers.

By assuming that $\mathbf{E}(\mathbf{r})$ is uniform inside the particle, we have:

$$
\begin{aligned}
\mathbf{E}(\mathbf{r}) & =\mathbf{E}_{0}(\mathbf{r})+k^{2}\left(\int_{V} \mathbf{G}\left(\mathbf{r}, \mathbf{r}^{\prime}, \omega\right) d^{3} \mathbf{r}^{\prime}\right) \epsilon_{0}(\boldsymbol{\epsilon}-\mathbf{I}) \mathbf{E}(\mathbf{r}) \\
& =\left[\mathbf{I}-k^{2}\langle\mathbf{G}\rangle_{V} V(\boldsymbol{\epsilon}-\mathbf{I})\right]^{-1} \mathbf{E}_{0}\left(\mathbf{r}_{p}\right)
\end{aligned}
$$

with:

$$
\langle\mathbf{G}\rangle_{V}=\frac{1}{V} \int_{V} \mathbf{G}\left(\mathbf{r}, \mathbf{r}^{\prime}, \omega\right) d^{3} \mathbf{r}^{\prime}
$$

We can express the limit of this quantities for small particle $(V \rightarrow 0)$ :

$\lim _{V \rightarrow 0}\langle\mathbf{G}\rangle_{V}=-\frac{\mathbf{I}}{3 k^{2}} V+\mathrm{i} \operatorname{Im}\left[\mathbf{G}_{0}\left(\mathbf{r}_{p}, \mathbf{r}_{p}, \omega\right)\right]+\mathbf{S}\left(\mathbf{r}_{p}, \mathbf{r}_{p}, \omega\right)$

Then, it comes easily that, for small particles:

$$
\begin{aligned}
\mathbf{E}(\mathbf{r})= & 3(\boldsymbol{\epsilon}+2 \mathbf{I})^{-1}\left\{\mathbf{I}-3 V k^{2}\left[\operatorname{iIm}\left\{\mathbf{G}_{0}\left(\mathbf{r}_{p}, \mathbf{r}_{p}, \omega\right)\right\}\right.\right. \\
& \left.\left.+\mathbf{S}\left(\mathbf{r}_{p}, \mathbf{r}_{p}, \omega\right)\right](\boldsymbol{\epsilon}-\mathbf{I})(\boldsymbol{\epsilon}+2 \mathbf{I})^{-1}\right\}^{-1} \mathbf{E}_{0}(\mathbf{r})
\end{aligned}
$$

Since the dipole moment of the particle is $\mathbf{p}=\epsilon_{0} V(\boldsymbol{\epsilon}-\mathbf{I}) \mathbf{E}(\mathbf{r})$ :

$$
\begin{aligned}
\mathbf{p}= & \epsilon_{0} \boldsymbol{\alpha}_{0}\left\{\mathbf{I}-3 V k^{2}\left[\operatorname{iIm}\left\{\mathbf{G}_{0}\left(\mathbf{r}_{p}, \mathbf{r}_{p}, \omega\right)\right\}\right.\right. \\
& \left.\left.+\mathbf{S}\left(\mathbf{r}_{p}, \mathbf{r}_{p}, \omega\right)\right] \boldsymbol{\alpha}_{0}\right\}^{-1} \mathbf{E}_{0}(\mathbf{r})
\end{aligned}
$$

where $\boldsymbol{\alpha}_{0}=3 V(\boldsymbol{\epsilon}-\mathbf{I})(\boldsymbol{\epsilon}+2 \mathbf{I})^{-1}$ is the static polarizability (ie. the polarizability when the radiation of the dipole outside in the external medium is neglected). The effective polarizability $\boldsymbol{\alpha}_{\text {eff }}$ defined in terms of exciting field as $\mathbf{p}=\epsilon_{0} \boldsymbol{\alpha}_{\text {eff }} \mathbf{E}_{0}(\mathbf{r})$ is given by :

$$
\boldsymbol{\alpha}_{\text {eff }}=\boldsymbol{\alpha}_{0}\left[\mathbf{I}-k^{2} \mathbf{G}_{p} \boldsymbol{\alpha}_{0}\right]^{-1}=\left[\mathbf{I}-k^{2} \boldsymbol{\alpha}_{0} \mathbf{G}_{p}(\omega)\right]^{-1} \boldsymbol{\alpha}_{0}
$$

where $\mathbf{G}_{p}=\operatorname{iIm}\left\{\mathbf{G}_{0}\left(\mathbf{r}_{p}, \mathbf{r}_{p}, \omega\right)\right\}+\mathbf{S}\left(\mathbf{r}_{p}, \mathbf{r}_{p}, \omega\right)=\operatorname{iIm}\left\{\mathbf{G}\left(\mathbf{r}_{p}\right.\right.$, $\left.\left.\mathbf{r}_{p}, \omega\right)\right\}+\operatorname{Re}\left\{\mathbf{S}\left(\mathbf{r}_{p}, \mathbf{r}_{p}, \omega\right)\right\}$. The last equality in (A7) can be easily verified without any assumptions on the commutability of the different tensors. *remi.carminati@espci.fr

${ }^{1}$ L. Novotny and B. Hecht, Principles of Nano-Optics (Cambridge University Press, Cambridge, 2006).

${ }^{2}$ W. L. Barnes, A. Dereux, and T. W. Ebbesen, Nature (London) 424, 824 (2003).

${ }^{3}$ E. Ozbay, Science 311, 189 (2006).

${ }^{4}$ M. I. Stockman, Phys. Today 64, 39 (2011).
${ }^{5}$ L. Novotny, Phys. Today 64, 47 (2011); L. Novotny and N. F. van Hulst, Nature Phot. 5, 83 (2011).

${ }^{6}$ A. V. Krasavin and N. I. Zheludev, Appl. Phys. Lett. 84, 1416 (2004).

${ }^{7}$ R. Espinola, T. Izuhara, M. Tsai, R. M. Osgood Jr., and H. Dötsch, Opt. Lett. 29, 941 (2004); H. Dötsch, N. Bahlmann, O. Zhuromskyy, M. Hammer, L. Wilkens, R. Gerhardt, P. Hertel, and A. F. Popkov, 
J. Opt. Soc. Am. B 22, 240 (2005); W. Van Parys, B. Moeyersoon, D. Van Thourhout, R. Baets, M. Vanwolleghem, B. Dagens, J. Decobert, O. Le Gouezigou, D. Make, R. Vanheertum, and L. Lagae, Appl. Phys. Lett. 88, 071115 (2006).

${ }^{8}$ B. Sepulveda, A. Calle, L. M. Lechuga, and G. Armelles, Opt. Lett. 8, 1085 (2006); D. Regatos, D. Fariña, A. Calle, A. Cebollada, B. Sepúlveda, G. Armelles, and L. M. Lechuga, J. Appl. Phys. 108, 054502 (2010).

${ }^{9}$ D. Martín-Becerra, J. B. González-Díaz, V. V. Temnov, A. Cebollada, G. Armelles, T. Thomay, A. Leitenstorfer, R. Bratschitsch, A. García-Martín, and M. U. González, Appl. Phys. Lett. 97, 183114 (2010); V. V. Temnov, G. Armelles, U. Woggon, D. Guzatov, A. Cebollada, A. García-Martín, J. M. García-Martín, T. Thomay, A. Leitenstorfer, and R. Bratschitsch, Nature Phot. 4, 107 (2010).

${ }^{10}$ Y. Hadad and B. Z. Steinberg, Phys. Rev. Lett. 105, 233904 (2010).

${ }^{11}$ R. Vincent and R. Carminati, Phys. Rev. B 83, 165426 (2011).

${ }^{12}$ F. A. Pinheiro, Phys. Rev. A 78, 023812 (2008).

${ }^{13}$ M. Inoue, K. Arai, T. Fujii, and M. Abe, J. Appl. Phys. 83, 6768 (1998); H. Kato, T. Matsushita, A. Takayama, M. Egawa, K. Nishimura, and M. Inoue, ibid. 93, 3906 (2003); Z. Wang and S. Fan, Opt. Lett. 30, 1989 (2005); W. Smigaj, J. Romero-Vivas, B. Gralak, L. Magdenko, B. Dagens, and M. Vanwolleghem, ibid. 35, 568 (2010).

${ }^{14}$ V. I. Safarov, V. A. Kosobukin, C. Hermann, G. Lampel, J. Peretti, and C. Marlière, Phys. Rev. Lett. 73, 3584 (1994).

${ }^{15}$ C. Hermann, V. A. Kosobukin, G. Lampel, J. Peretti, V. I. Safarov, and P. Bertrand, Phys. Rev. B 64, 235422 (2001); J. B. GonzálezDíaz, A. García-Martín, G. Armelles, J. M. García-Martín, C. Clavero, A. Cebollada, R. A. Lukaszew, J. R. Skuza, D. P. Kumah, and R. Clarke, ibid. 76, 153402 (2007); E. Ferreiro-Vila, J. B. Gonzalez-Diaz, R. Fermento, M. U. Gonzaĺez, A. García-Martín, J. M. García-Martín, A. Cebollada, G. Armelles, D. MenesesRodríguez, and E. M. Sandoval, ibid. 80, 125132 (2009).
${ }^{16}$ J. F. Torrado, J. B. González-Díaz, M. U. González, A. GarcíaMartín, and G. Armelles, Opt. Express 18, 15635 (2010); E. T. Papaioannou, V. Kapaklis, P. Patoka, M. Giersig, P. Fumagalli, A. García-Martín, E. Ferreiro-Vila, and G. Ctistis, Phys. Rev. B 81, 054424 (2010).

${ }^{17}$ D. Van Labeke and D. Barchiesi, J. Opt. Soc. Am. A 9, 732 (1992); R. Carminati and J.-J. Greffet, ibid. 12, 2716 (1995); J.-J. Greffet and R. Carminati, Prog. Surf. Sci. 56, 133 (1997).

${ }^{18}$ A. Madrazo, R. Carminati, M. Nieto-Vesperinas, and J.-J. Greffet, J. Opt. Soc. Am. A 15, 109 (1998).

${ }^{19}$ A. B. Evlyukhin and S. I. Bozhevolnyi, Phys. Rev. B 71, 134304 (2005); A. B. Evlyukhin, G. Brucoli, L. Martín-Moreno, S. I. Bozhevolnyi and F. J. García-Vidal, ibid. 76, 075426 (2007).

${ }^{20}$ R. P. Hunt, J. Appl. Phys. 38, 1652 (1967); J. Zak, E. R. Moog, C. Liu, and S. D. Bader, J. Magn. Magn. Mater. 89, 107 (1990).

${ }^{21}$ D. Bedeaux and P. Mazur, Physica 67, 23 (1973); V. V. Varadan, A. Lakhtakia, and V. K. Varadan, IEEE Trans. Antennas Propag. 37, 800 (1989); A. Lakhtakia, Astrophys. J. 394, 494 (1992); A. H. Sihvola, Opt. Lett. 19, 430 (1994).

${ }^{22}$ S. Albaladejo, R. Gómez-Medina, L. Froufe-Pérez, H. Marinchio, R. Carminati, G. Armelles, J. Torrado, A. García-Martín, and J. Sáenz, Opt. Express 18, 3556 (2010).

${ }^{23}$ B. Sepúlveda, J. B. González-Diaz, A. Garcia-Martín, L. M. Lechuga, and G. Armelles, Phys. Rev. Lett. 104, 147401 (2010).

${ }^{24}$ F. Pincemin, A. Sentenac, and J.-J. Greffet, J. Opt. Soc. Am. A 11, 1117 (1994).

${ }^{25}$ The dielectric functions of $\mathrm{Au}$ and $\mathrm{Ag}$ are taken from P. B. Johnson and R. W. Christy, Phys. Rev. B 6, 4370 (1972). The dielectric function of Co is taken from E. Ferreiro-Vila et al., ibid. 80, 125312 (2009).

${ }^{26}$ R. Carminati, J.-J. Greffet, C. Henkel, and J. M. Vigoureux, Opt. Commun. 261, 368 (2006).

${ }^{27}$ J. E. Sipe, J. Opt. Soc. Am. B 4, 481 (1987). 